\title{
The role of social media marketing in consumer behaviour
}

\author{
Sayabek Ziyadin ${ }^{1 *}$, Raigul Doszhan ${ }^{1}$, Alex Borodin ${ }^{2}$, Aizhan Omarova ${ }^{1}$, and Aigerim Ilyas ${ }^{1}$ \\ ${ }^{1}$ Al-Farabi Kazakh National University, 050040, 71, al-Farabi Ave, Almaty, Kazakhstan \\ ${ }^{2}$ Plekhanov Russian University of Economics, 117997, 36, Stremyanny lane, Moscow, Russia
}

\begin{abstract}
This article looks at recently published research on social media consumers. Five topics are highlighted: consumer digital culture, responses to digital advertising, the impact of social media on consumer behavior, mobile environments, and online rumors (WOM). The articles examines how consumers experience, are influenced and are influenced by the digital environment they are in as part of their daily lives. Much remains to be understood, and existing knowledge tends to focus disproportionately on WOM, which is only part of the digital consumer experience. Several avenues for future research have been proposed to encourage researchers to consider a wider range of phenomena.
\end{abstract}

\section{Introduction}

The use of the Internet, social media, mobile applications and other digital communication technologies has become part of billions of people's daily lives. For example, the current level of Internet use among adults is about $87 \%$ and closer to $100 \%$ for demographic groups, such as adults with higher education and higher income. Young people - the next generation of mass consumers - have the same high levels. People are also spending more and more time on the Internet. In the UK, for example, the number of hours spent online by adults has more than doubled over the past decade and now averages 20.5 hours per week. Social media support part of this growth: around the world, more than 2 billion people now use social media, and Facebook alone now has about 1 billion active users per day.

Social media have been largely implemented as an effective mechanism that promotes the marketing goals and strategies of firms, especially in aspects related to customer participation, customer relationship management and communications. For example, social media can strategically improve two-way communication between firms and customers and, accordingly, connect more customers to organizations. This will be in addition to the ability of social media to present content that is published visually, verbally or textually, or using a combination of text, visual and verbal content. In different contexts, firms have been eagerly anticipating the use of social media in many aspects of their customer

\footnotetext{
"Corresponding author: sayabekz@gmail.com
} 
interactions, such as facilitating information retrieval, interactivity, promotion and improving customer purchasing behaviour.

People are showing themselves more and more in social media. This is done for many purposes, including their role as consumers, as they seek information about products, buy and consume them, and communicate with others about their experiences. Marketers have responded to this fundamental shift by increasing the use of digital marketing channels. In fact, by 2017 , about a third of global advertising spending will be on digital channels. Thus, in the future, consumer marketing will focus on the digital environment, especially social media and mobile devices. Therefore, consumer research needs to examine and understand consumer behaviour in the digital environment. This has been the case over the past decade, and more research has focused on digital consumer behaviour issues. More research is still emerging in the literature, however, of course - especially given the ever-changing nature of social media and mobile environments in which consumers are located and interact with brands and each other. This article attempts to summarize the latest developments on these issues in the literature on consumer behaviour and psychology, while hoping to stimulate new relevant research.

\section{Literature review}

Social media marketing is the process of drawing attention to the brand through social media. Also, social media marketing is aimed at creating content that would attract the attention of users and encourage them to share it with their readers.

Social media provide people with common interests with a virtual space to share and discuss ideas. According to Raacke and Bonds - Raacke, social networks allow users to create a community through constant communication. Long-term information sharing and growth contribute to the development of loyal social relationships [1]. According to K.Burton, the amount of information published by the user on social networks is positively correlated with the number of subscribers the user has. It is also noted that in the online environment, people like to express themselves, share ideas, contribute, create and join communities of people to meet the needs of belonging, be socially connected or simply enjoy communicating with other like-minded people. According to D. Falls, social media marketing is a type of internet marketing that is used in various social media to achieve the goals of marketing communication and branding [3]. Social media marketing is mainly aimed at posting and sharing different types of content to achieve marketing goals. Social networks, on the other hand, allow consumers to interact, and they are a very effective Internet - a tool for information exchange between people.

Social networks are the main type of social media. Social networks are platforms for communication and interaction that contribute to increasing trust between communities. According to Y.Akrimi, any website or online platform that allows users to share their opinions, views, content, and encourages interaction and community building can be classified as a social network [4]. The most popular social networks are: Facebook, VKontakte, Instagram, YouTube, Twitter, Odnoklassniki, as well as messengers WhatsApp, Telegram. Social networks have significantly changed the way information is disseminated, making it a very easy process to share. It is also worth noting that the unique opportunities and tools of social media and their huge popularity influenced marketing and advertising. According to W. G.Mangold, social media also influence consumer behavior: how they consume information as post-purchase behavior, such as post-purchase behavior, for example, writing reviews or expressing their dissatisfaction and dissatisfaction with the experience of using the product [5].

A.M.Kaplan defines social media as "a group of Internet applications based on the ideological and technological foundations of the Web 2.0 platform and allowing the 
creation and sharing of user-generated content. Scientists Kaplan and Heinlein also note that social media have many advantages, as they help to establish strong links between companies and consumers, develop their relationships in a timely manner and, most often, at low cost [6]. According to M.Laroche, important functions of social media are to influence the perception, attitude and final behavior of consumers. It is also noted that in the online environment people like to express themselves, share ideas, contribute, create and join communities of people to meet the needs of belonging, be socially connected or simply enjoy communicating with other like-minded people [7].

According to S. Ziyadin, social networks are channels of information transfer, the way we share our lives through pictures, words, videos [8].

According to D. Mersey, social media provide companies with the opportunity to interact with potential and existing consumers, creating a deeper sense of intimacy with customers and building more meaningful relationships with consumers [9]. This is particularly important in today's business environment, where consumer confidence can be lost due to the slightest mistake, which can lead to the spread of negative feedback and information about a particular product, service, brand or company. Therefore, many enterprises are beginning to notice the real power of social networks and social media.

According to L.Spiller, social media marketing is the use of technologies, channels and software for social networks, which consists in creating, exchanging, delivering and exchanging offers that have value for the stakeholders of the organization [10].

The concept of "consumer" is very closely related to the concept of "consumer behavior". F. Kotler defines: "Consumer behaviour of an individual consumer is the behaviour of an end user who buys services and goods for personal use. Together, consumers create a consumer market" [11].

\section{Methodologies}

A comparison was made for the analysis using statistics on the use of social networks. Expert and analytical publications and other open sources of information were used as sources of secondary information.

Five different research topics are covered by the recent consumer research on social media. Five themes: consumer digital culture, advertising, digital environment, mobile and online WOM. The most popular topics were online WOM, which covered almost half of the articles, and advertising, represented by just over a quarter of the articles.

\subsection{Consumer digital culture}

The study of consumer digital culture takes a rather deep look at the digital environment in which consumers live. A key aspect of this work was understanding how consumer identities and the concept of I apply to the digital worlds [12]. Belk has expanded his previous work on the "extended self" to include the digital environment in which consumers now live, which is an important part of the development of the theory, as it addresses concepts such as the ability of consumers to have multiple selfs because of multiple online identities. Belk also offers many areas for future research. Other studies on this topic have examined more specific phenomena. McQuarrie has focused on fashion blogs as a way to document the "megaphone effect", which is an opportunity for regular consumers to access a wide audience through social media [13]. This is an important effect, and they discussed how bloggers build an audience and accumulate social (or cultural) capital by demonstrating good taste. This is true in certain contexts, but it is important to understand consumer behaviour in creating content on social media in general, as the signalling of positive personalities is likely to be a common motivation to post certain 
things on sites such as Facebook. Together these articles make an important conceptual contribution to the way we see consumers in the digital world, in particular because of the broader concept of what it means to be a consumer in today's digital world.

\subsection{Advertising}

Social media marketing is the most economical way to advertise any product. Creating a new profile and subscription are free in almost all social networks. Awareness of the advertised brand is increasing, as the company interacts with a wide audience. To get started, you need to create profiles in social networks and start interacting with them. Simply communicating with potential customers will increase brand recognition and start building a business reputation. Each message that will be disseminated will be presented by a new group of people, which can lead to them becoming potential customers, and the more people who know about the business, the better.

Thus, we can say that social media advertising works well when it comes to reaching a wide range of people, as the effect of "sarafan radio". There is no doubt that simply having a page on social media will make a brand recognizable, and if used regularly, it can create a wide audience for business. Advertising in social media gives a lot of control over the target audience.

Digital advertising is a major topic in the marketing literature and, with regard to consumer behaviour, it looks at how consumers react to various aspects of digital advertising. J.H.Schumann has considered how to overcome negative reactions to personalization through normative reciprocity appeals (instead of utility appeals) [14]. Lambrecht studied ad retargeting when personalized recommendations based on previous browsing histories are made when the consumer returns to the website [15]. Negative responses to retargeting occur, but this is mitigated when consumer preferences become more precise. He found that personalized ads on a website are more favorably received when consumers better perceive control over personal information used for personalization, which is directly consistent with the literature on psychological reactions and offers a theoretical way forward.

X. Luo examined the drivers of popularity of group shopping advertising (i.e., "daily transactions"), finding that the main factor in popularity is social influence [16]. He studied the answers to search engine advertising and found that when consumers look for less popular keywords, it becomes more time-consuming to find them. Puccinelli considered how emotions in content (e.g. TV shows) combined with advertising energy levels affect consumer responses, and found that the affective relationship between content and advertising matters, so that when consumers experience 'decontaminating' emotions (e.g. sadness, sadness), it is more difficult to look at vigorous advertising [17]. He looked at how digital media and search advertisements stimulate online shopping for retailers and found that digital advertisements are more effective than offline advertisements in managing online consumer behaviour.

\subsection{Impact of digital environments}

In recent years, the topic of how the social networking environment influences consumer behaviour has still emerged. It is interesting to see how different information and social characteristics of the social environment, such as the impact of other consumers' opinions or choices (e.g. bids on online auctions), or even the lives of friends through social networks, can affect subsequent behaviour. For example, with regard to the integral consequences for the environment, Lamberton has looked at learning from others in the digital environment and identified how such observations can influence individuals' 
decisions in these environments, as well as the conclusions they draw about others [18]. Scientists have examined how the environment reacts to how the use of Facebook has affected self-control. They found that when in contact with closer friends on Facebook, consumers subsequently showed lower levels of self-control when choosing, for example, healthy behaviour (e.g., choosing a healthier snack instead of an unhealthy option). However, this was only for people who knew their closest friends on Facebook.

Consumer behaviour in mobile settings is becoming more and more important as consumers use mobile devices more often. This is particularly interesting in the context of shopping. In the store, scientists have studied how consumers react to mobile offers in physical stores, seeing how mobile coupons can change the way consumers go. In an online store, consumers focus on shopping on mobile devices (e.g. tablets) and in particular on how touching products (instead of clicking on them) can enhance their sense of belonging and endowment. This is an interesting contribution because work on how consumers physically interact with mobile devices and how this affects decision-making is scarce [19]. This is seen as a mobile ad display - which is very small and has very little (if any) information - to consumers of branded attitudes and purchasing intentions. They have found that in many product categories mobile media advertisements have no effect, but they raise attitudes and intentions for utilitarian products with high levels of involvement.

\subsection{Online WOM}

Consumers rely on information from social sources. A number of subtopics have been covered recently. The imagery language in online surveys has had a positive impact on consumer attitudes and the choice of hedonic products. S.Moore reviewed the explanatory language in the online surveys, finding that what consumers attributed to actions or reactions had an impact on perceived utility [20]. These, often small in resources and staff, enterprises have to quickly develop, introduce new marketing channels and skills and be innovative so that budgets go further [21]. He looked at the negative WOM, finding that the use of mitigating language in expressing negative opinions increases the credibility and attractiveness of the reviewer. T. Tang considered two kinds of neutral language, mixed (positive and negative) and indifferent, showing that neutral WOM enhances the direct influence of positive and negative WOM on a purchase if it is mixed. Another important topic recently discussed is the difference between online and WOM offline [22]. OnlineWOM is defined by the social and functional characteristics of the brand, while offlineWOM is defined by the emotional characteristics of the brand, based on a large set of data about the WOM brand online and offline settings. Accepting a narrower but nevertheless important viewpoint, researchers found differences between WOM's social media transmission (e.g. Facebook) and offline (personal), showing that consumers are less likely to transmit WOM in social media because of higher perceived social risk.

\section{Discussion and results}

The number of social network users grows day by day. Compared to 2014, the number of Internet users worldwide has increased by more than 1.9 billion, or $75 \%$ in five years. (Fig. $1)$. 


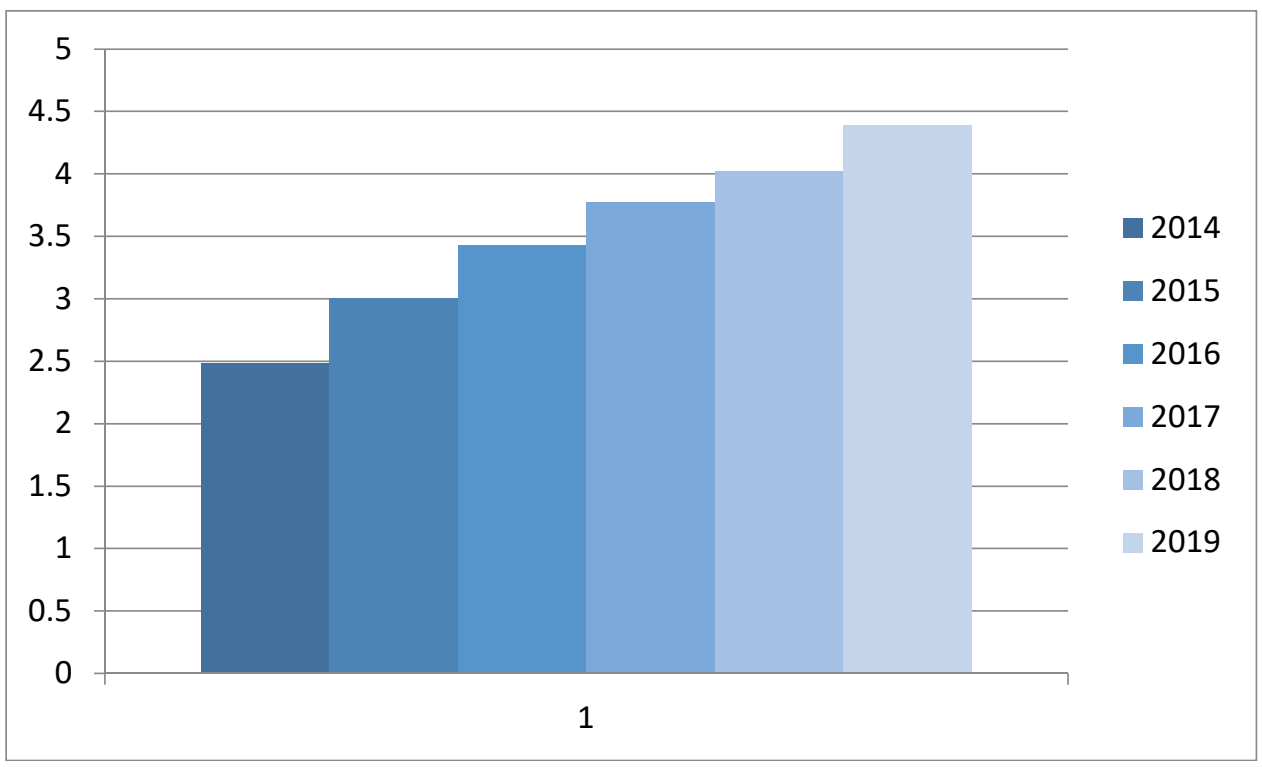

Fig. 1. Internet audience growth dynamics from 2014 to 2019 (billion people).

According to the International Telecommunication Union, it took 16 years to reach the first billion Internet users. The second billion joined the network in just 6 years. Today, the Internet is growing at a rate of 1 billion new users in 2.7 years.

The dynamics of social media audience growth over the last 5 years is even more impressive than the data on the use of the Internet over the same period - since 2014 the total number of social media users in the world has almost doubled. This year's figure -3.49 billion social media users - is 2 billion more than in January 2014 (Figure 2).

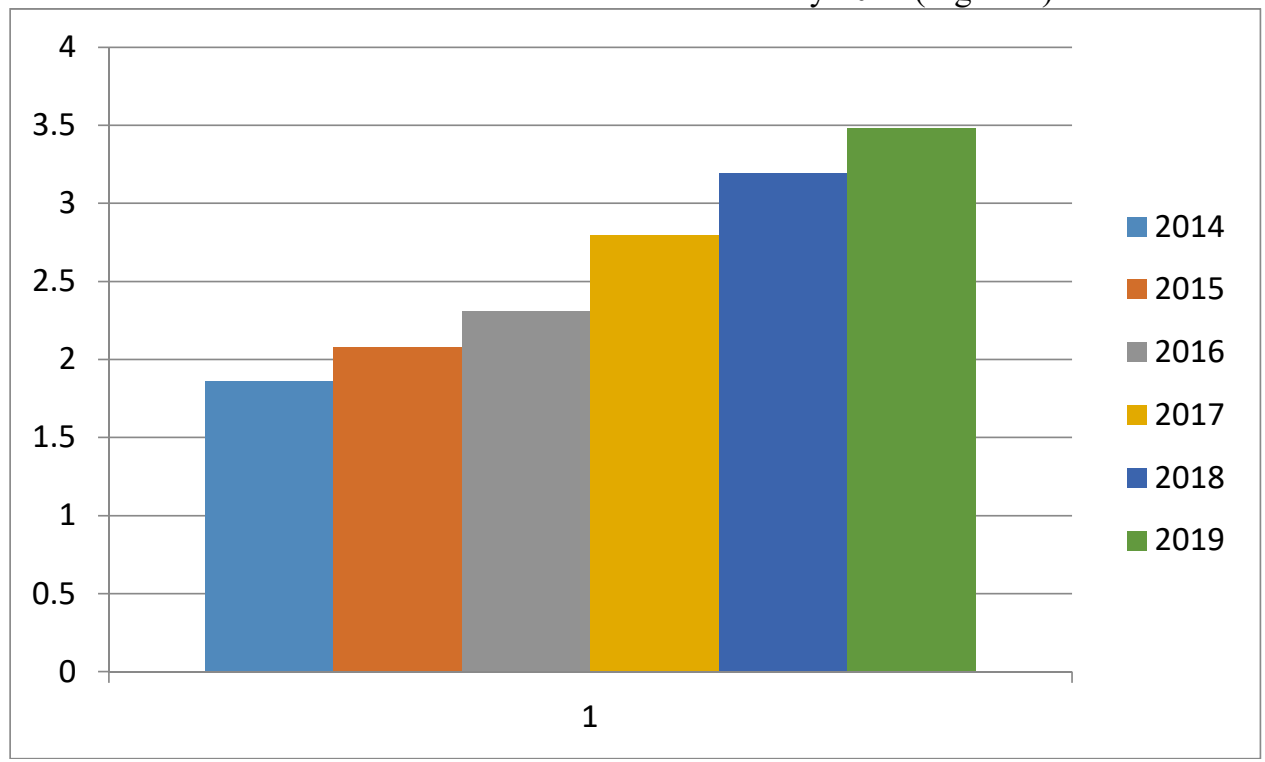

Fig. 2. Social networks growth dynamics from 2014 to 2019 (billion people). 


\subsection{Behaviour of social network users in 2019}

The amount of time people spend on social media has increased again this year, albeit insignificantly. GlobalWebIndex reports that today the average user (in the world) spends 2 hours and 16 minutes every day on social platforms, and in 2018 this indicator was 2 hours and 15 minutes. This is about a third of the total time on the Internet and a seventh of the total time of waking up.

It is worth noting that in different cultures, the time spent on social networks is significantly different - in Japan, for example, Internet users spend on social networks only 36 minutes a day. On the other side of the spectrum are Filipinos, who (as last year) are the most active on social media platforms. This year, the average figure for the Philippines is 4 hours and 12 minutes, an increase of 15 minutes per day (6\%) compared to last year.

\subsection{Growth of Instagram popularity}

Search by the term "social media" in any Internet search engine gives results: popular "Facebook", "Twitter", "Instagram" and "YouTube", "Vkontakte" and others. Marketers all over the world agree that Instagram will be the best platform for brands in 2019. Despite some problems in management in 2018, Instagram has achieved serious success in the last 12 months. Back in June, the company announced that it had overcome the mark of 1 billion "active accounts". Although it was later explained that this number does not reflect the number of unique users, it is equally impressive. The latest data on the available advertising audience shows that these high figures are maintained in 2019 - over the past 3 months, the number of active users has increased by more than $4 \%$ over the past 3 months, reaching 895 million worldwide. Instagram audience may be half as small as Facebook, but over the past three months Instagram has added more than twice as many new users as Facebook. The platform increased its global advertising audience by 38 million new users in the 4th quarter of 2018, while Facebook grew by only 18 million new users in the same period of time. However, Instagram's popularity with marketers is not only due to its growing user base, but also due to the fact that this social network boasts a balanced audience profile. The ratio of men and women in the global audience of Instagram is about 50:50, and although the average user here is still younger than in Facebook, Instagram is more common in the audience aged 18 to 34 years than the main brainchild of Zuckerberg (though the total number of users of this age there is less).

During 2018 Instagram demonstrated impressive growth all over the world, and now this social network has become a global one. Instagram's advertising audience has outgrown the Facebook advertising audience in 20 countries, and this number is expected to increase in 2019.

The data presented show global revenues from social media advertising for the period from 2015 to 2018. According to the source, in 2018 revenue will amount to 41.6 billion US dollars. According to the source, in 2018 revenue will amount to $\$ 41.6$ billion, compared to $\$ 17.89$ billion in 2015 (Fig. 3). According to 2017 estimates, Facebook and YouTube together account for nearly three-quarters of the social media advertising market. A recent study by IAB (Internet Advertising Bureau) shows that in the first half of 2018, social media spending grew by $53 \%$, with a total contribution of USD 242.5 million. 
Table 1. Potential advertising coverage in Instagram, by age group and gender.

\begin{tabular}{|c|c|c|c|c|c|}
\hline Age & $\begin{array}{c}\text { The whole } \\
\text { auditory }\end{array}$ & $\begin{array}{c}\text { Women } \\
\text { Total }\end{array}$ & $\begin{array}{c}\text { The } \\
\text { percentage of } \\
\text { women }\end{array}$ & $\begin{array}{c}\text { Men } \\
\text { total }\end{array}$ & $\begin{array}{c}\text { The } \\
\text { percentage of } \\
\text { men }\end{array}$ \\
\hline $13-17$ & 57000000 & 30000000 & $3 \%$ & 27000000 & $3 \%$ \\
\hline $18-24$ & 280400000 & 130200000 & $15 \%$ & 150200000 & $17 \%$ \\
\hline $25-34$ & 290400000 & 142200000 & $16 \%$ & 150200000 & $17 \%$ \\
\hline $35-44$ & 142200000 & 76100000 & $9 \%$ & 66100000 & $7 \%$ \\
\hline $45-54$ & 73100000 & 41100000 & $5 \%$ & 32000000 & $4 \%$ \\
\hline $56-64$ & 32000000 & 19000000 & $2 \%$ & 13000000 & $1 \%$ \\
\hline $65+$ & 19800000 & 11000000 & $1 \%$ & 8800000 & $1 \%$ \\
\hline Всего & 894900000 & 447600000 & $50.3 \%$ & 447300000 & $49.7 \%$ \\
\hline
\end{tabular}

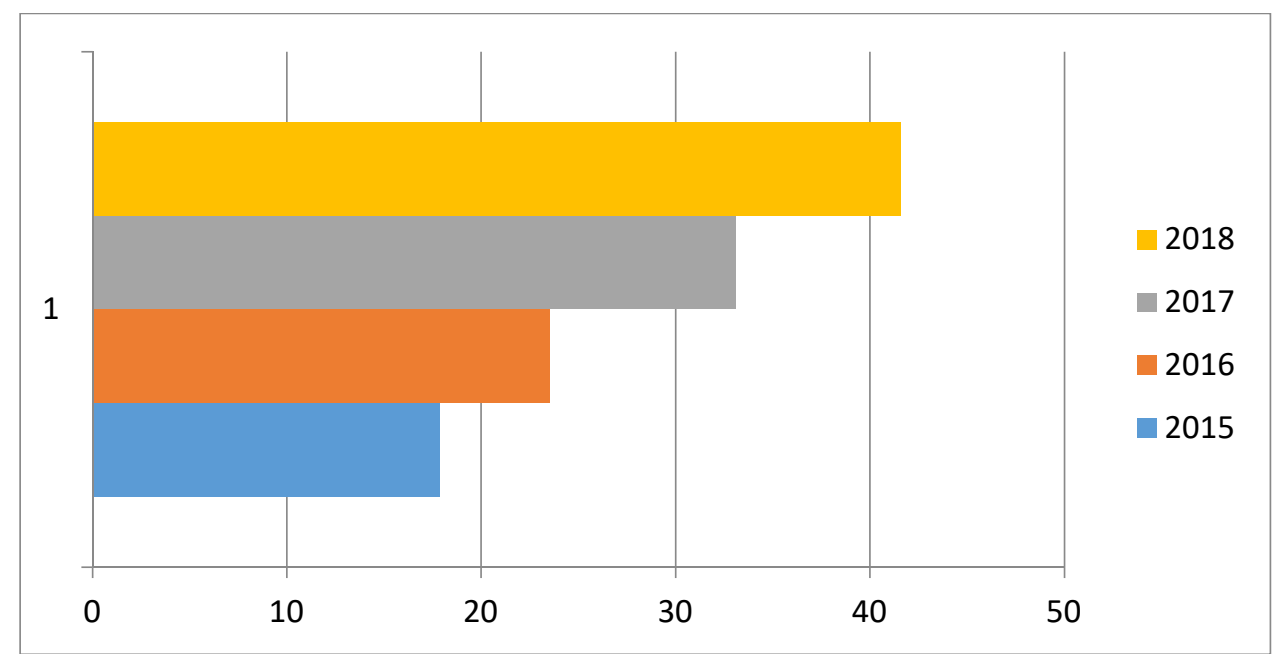

Fig. 3. Revenue from advertising in social networks for the period from 2015 to 2018 (in USD billion).

Analyzing the information, we can say that, first of all, such an investment amount suggests that this is not a fleeting fad, and secondly, many people want to promote their product or service in their favorite social network. The purpose of advertising is to win the market through brand loyalty. Not surprisingly, companies around the world are increasingly investing in social media marketing to achieve the company's goals. Also, if a company wants to enter completely different markets, such as Spain, it can place advertising in the popular social network of the country, thereby effectively presenting its products to the target audience. If the purpose of the business is to attract as many customers as possible, the presence in social media is necessary. Social media marketing is the most economical way to advertise any product. Creation of a new profile and subscription are free of charge in almost all social networks. Awareness of the advertised brand is increased, as the company interacts with a wide audience. 


\section{Conclusion}

Thus, we believe that social media advertising, while not devoid of shortcomings, is still a great way to increase brand loyalty, and in the digital economy, especially as it allows you to interact directly with potential customers on a regular basis. Social media demand: the information field of people and enterprises is expanding significantly, the costs of searching, exchanging and storing information will decrease, as well as the role of information as a resource in the economic management system will be strengthened, it will help the company to reach a larger number of consumers, increase the volume of income and increase the competitiveness of the enterprise. Application of marketing technologies allows to carry out promotion and realization of common resources of the enterprises, including goods and services and to choose effective methods of promotion for each product. It also helps to increase the efficiency of their activities. The use of social media in marketing will increase brand loyalty, directly, and on a regular basis, to interact with potential customers in the digital economy, so many companies understand the importance and necessity of using social media in marketing.

\section{References}

1. J. Raacke, J. Bonds-Raacke, Cyberpsych. \& Beh., T. 11. - №. 2., 169-174 (2008)

2. K. Burton, (ICWSM (2009)

4. Y. Akrimi. R. Khemakhem, J. Mark. Res. \& C. S., T.2012, 1 (2012)

5. W. G. Mangold, D. J. Faulds, Bus. Horiz., 52(4), 357-365 (2009)

6. A. M. Kaplan, M. Haenlein, Bus. Horiz. 53(1), 59-68 (2010)

7. M. Laroche, Compu. Hum. Be., 28(5), 1755-1767 (2012)

8. S., Ziyadin, A. Serikbek, Scientific Conference "Digital Transformation of the Economy: Challenges, Trends, New Opportunities" 290-297 (2019)

9. Davis Mersey, E. C. Malthouse, B. J. Calder, J. Med. Bus. St., 7(2), 39-56 (2010)

10. L. Spiller, T. Tuten, J. Mark. Ed. 10 (2015)

11. A.V. Bataev, A.A. Gorovoy, A.B. Mottaeva, IBIMA 2018 - Vision 2020: Sustainable

Economic Development and Application of Innovation Management from Regional expansion to Global Growth, 102-114 (2018)

12. R. Belk, Curr. Opin. Psych., 10, 50-54 (2016)

13. E. F. McQuarrie, J. Miller, B. J. Phillips, J.Con. Res., 40(1), 136-158 (2012)

14. J. H. Schumann, F. Wangenheim, N. Groene, J. Mark., 78(1), 59-75 (2014)

15. A. Lambrecht, C. Tucker, J. Market. Res., 50(5), 561-576 (2013)

16. X. Luo, J. Market, 78(2), 20-33 (2014)

17. N. M. Puccinelli, K. Wilcox, D. Grewal, J. Market., T. 79. №. 2., 1-18 (2015)

18. C. P. Lamberton, R. W. Naylor, K. L. Haws, J. Cons. Psych.., T. 23. №. 1 74-89 (2013)

19 Y. Bart, A. T. Stephen, M. Sarvary, J. Marke. Res., T. 51. №. 3., 270-285 (2014)

20. S. G. Moore, J. C. Resea., T. 42. - №. 1., 30-44 (2015)

21. Ziyadin Sayabek, Dauliyeva Galiya, Kalymbekova Zhanna and Turlybekova Asel, IBIMA, 2065-2070 (2017)

22. T. Tang, E. Fang, F. Wang, J. Market., T. 78. №. 4., 41-58 (2014) 